\title{
Síncope como manifestación clínica del bloqueo aurículo- ventricular paroxístico y respuesta al implante de marcapasos bicameral
}

\section{Syncope as a clinical manifestation of paroxysmal atrioventricular block and response to bicameral pacemaker implantation}

Helber Gonzalo López-Patiño ${ }^{1 凶} \underline{\text { ORCID }}$, María del Mar Serna-Posada ${ }^{2 \bowtie} \underline{\text { ORCID }}$, Laura Duque-González ${ }^{3 凶} \underline{\mathrm{ORCID}}$, William Uribe-Arango $4 \llbracket \underline{\mathrm{ORCID}}$, Mauricio Duque-Ramírez ${ }^{4 \bowtie} \underline{\mathrm{ORCID}}$

\section{${ }^{1}$ Especialista en Cardiología, Hospital General de Medellín.}

${ }^{2}$ Médica de enlace en Cardiología, CES Cardiología. Grupo de investigación: Enfermedades del corazón CES.

${ }^{3}$ Especialista en Cardiología, CES Cardiología. Grupo de investigación: Enfermedades del corazón CES.

${ }^{4}$ Especialista en Cardiología y Electrofisiología, CES Cardiología. Grupo de investigación: Enfermedades del corazón CES

Fecha correspondencia: Recibido: mayo 27 de 2020. Revisado: abril 22 de 2021. Aceptado: mayo 13 de 2021.

Forma de citar:

López-Patiño HG, Serna-Posada $M$ del $M$, Duque-González $L$, Uribe-Arango W, Duque-Ramírez M. Síncope como manifestación clínica del bloqueo aurículoventricular paroxístico y respuesta al implante de marcapasos bicameral. Rev CES Med. 2021; 35(2): 156-164. 10.21615/cesmedicina.5672

\section{Open access}

\section{(C) Derecho de autor}

Licencia creative commons

Ética de publicaciones

Revisión por pares

Gestión por Open Journal System DOI: http://dx.doi.org/10.21615/ cesmedicina. 5672

ISSNe 2215-9177

ISSN 0120-8705

Publica con nosotros

\section{Resumen}

El síncope es el principal síntoma que presentan los pacientes con bloqueo aurículo-ventricular completo paroxístico y puede obedecer a una serie de etiologías intrínsecas o extrínsecas. Los bloqueos son causa de disfunción importante, con alta carga de morbilidad, incluso en pacientes sin cardiopatía isquémica o anomalía estructural de base. Se presenta el caso de una paciente con corazón estructuralmente normal, quien ingresó para estudio de síncope y durante el monitoreo Holter de 24 horas se documentó bloqueo aurículo-ventricular completo paroxístico, por lo cual se procedió al implante de un marcapasos bicameral, con muy buena respuesta a la intervención. Los bloqueos aurículo-ventricular completos pueden ser paroxísticos o permanentes y la única forma de diferenciarlos son los hallazgos electrocardiográficos. Es muy importante realizar el diagnóstico de bloqueo aurículo-ventricular completo, bien sea paroxístico o definitivo, porque el único tratamiento es el implante de un dispositivo de estimulación eléctrica cardíaca.

Palabras clave: Bloqueo Aurículo-ventricular; Electrocardiograma; Marcapasos; Síncope. 


\section{Abstract}

Syncope is the main symptom presented by patients with paroxysmal complete atrioventricular block, and it may be due to several intrinsic or extrinsic etiologies. AV blocks are a major cause of dysfunction, with a high burden of disease, even in patients without ischemic heart disease or underlying structural abnormality. The case of a patient with a structurally normal heart is presented, who was admitted for a syncope study and during a 24-hour Holter monitoring, a paroxysmal complete AV block was documented, which led to the implantation of a bicameral pacemaker and had a very good response to the procedure. Complete AV blocks can be paroxysmal or permanent, and the only way to differentiate them is by electrocardiographic findings. It is very important to make the diagnosis of complete AV block, either paroxysmal or permanent, because the only treatment is the implantation of a cardiovascular implantable electronic device.

Keywords: Atrioventricular block; Electrocardiography; Pacemaker; Syncope.

\section{Introducción}

El síncope se define como la pérdida completa y transitoria del estado de conciencia, debida a una hipoperfusión cerebral, asociada con pérdida del tono postural y con una recuperación espontánea e inmediata ${ }^{(1)}$. Este se clasifica en tres grandes grupos según su etiología: neuralmente mediado o reflejo, secundario a hipotensión ortostática o de origen cardiogénico (figura 1) $)^{(2)}$.

Es un síntoma común que representa un porcentaje importante de consultas por urgencias e ingresos hospitalarios. A pesar de que en la mayoría de los casos, la etiología es benigna, causa gran impacto e incertidumbre en quienes lo padecen, lo cual lo hace un motivo de consulta frecuente que requiere de un diagnóstico preciso y de un manejo oportuno y adecuado ${ }^{(3,4)}$.

Las principales causas son: síncope reflejo (neuralmente mediado), síncope por hipotensión ortostática y síncope de origen cardíaco(2). El síncope de origen cardíaco puede ser debido a enfermedad cardíaca estructural como la estenosis aórtica o a arritmias, que pueden ser taqui o bradiarritmias ${ }^{(5)}$. Estas últimas incluyen la disfunción del nodo sinusal y los trastornos de la conducción aurículo-ventricular (AV), como el bloqueo AV de segundo grado Mobitz II y el bloqueo AV completo paroxístico/intermitente o permanente ${ }^{(1,6)}$. El síncope es el principal síntoma que presentan los pacientes con bloqueo aurículo-ventricular completo paroxístico. 
Mayo - agosto de 2021

Existen tres causas de bloqueo aurículo-ventricular paroxístico: las enfermedades intrínsecas del sistema de conducción, el aumento del tono vagal ${ }^{(7,8)}$, y el más recientemente descrito: el idiopático de comportamiento paroxístico ${ }^{(9,10)}$. Se presenta el caso de una paciente con historia de múltiples episodios de síncope, sin enfermedad cardíaca estructural de base, y en quien se encontró como causa más probable de estos un bloqueo AV paroxístico idiopático.

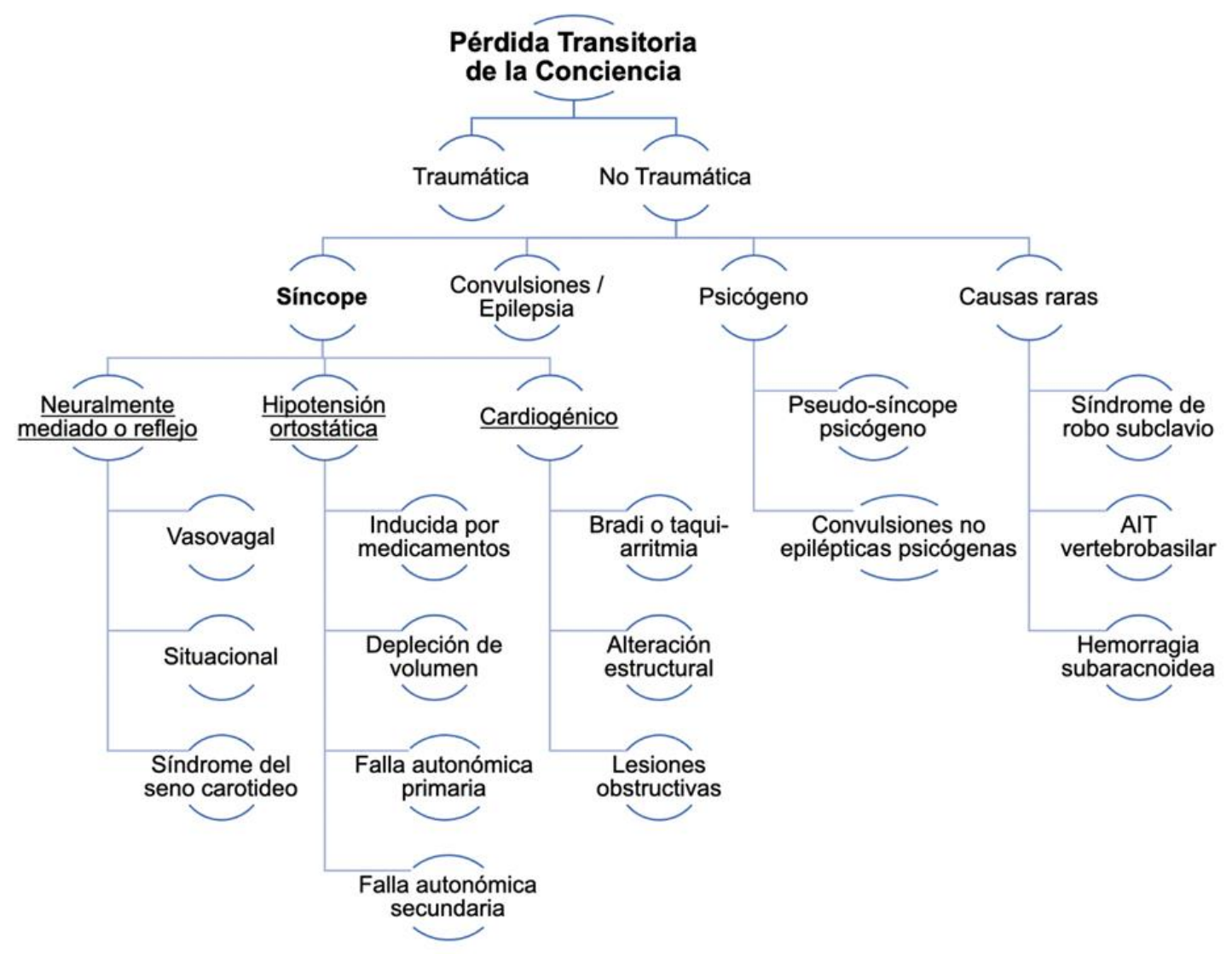

Figura 1. Enfoque de la pérdida transitoria de la conciencia y clasificación del síncope, con sus diagnósticos diferenciales más importantes según las guías europeas de cardiología 2018 (AIT: ataque isquémico transitorio).

\section{Reporte del caso}

Mujer de 42 años remitida a consulta de Electrofisiología por un cuadro clínico consistente en múltiples episodios sincopales de tres meses de evolución, algunos con trauma importante, sin clínica premonitoria, en ausencia de actividad física y en ocasiones con dolor precordial de tipo opresivo irradiado al miembro superior izquierdo y en quien se descartó síndrome coronario agudo con biomarcadores cardíacos. Se le realizó un monitoreo Holter 24 horas en el que se documentó un bloqueo aurículo-ventricular completo paroxístico (figura 2). 


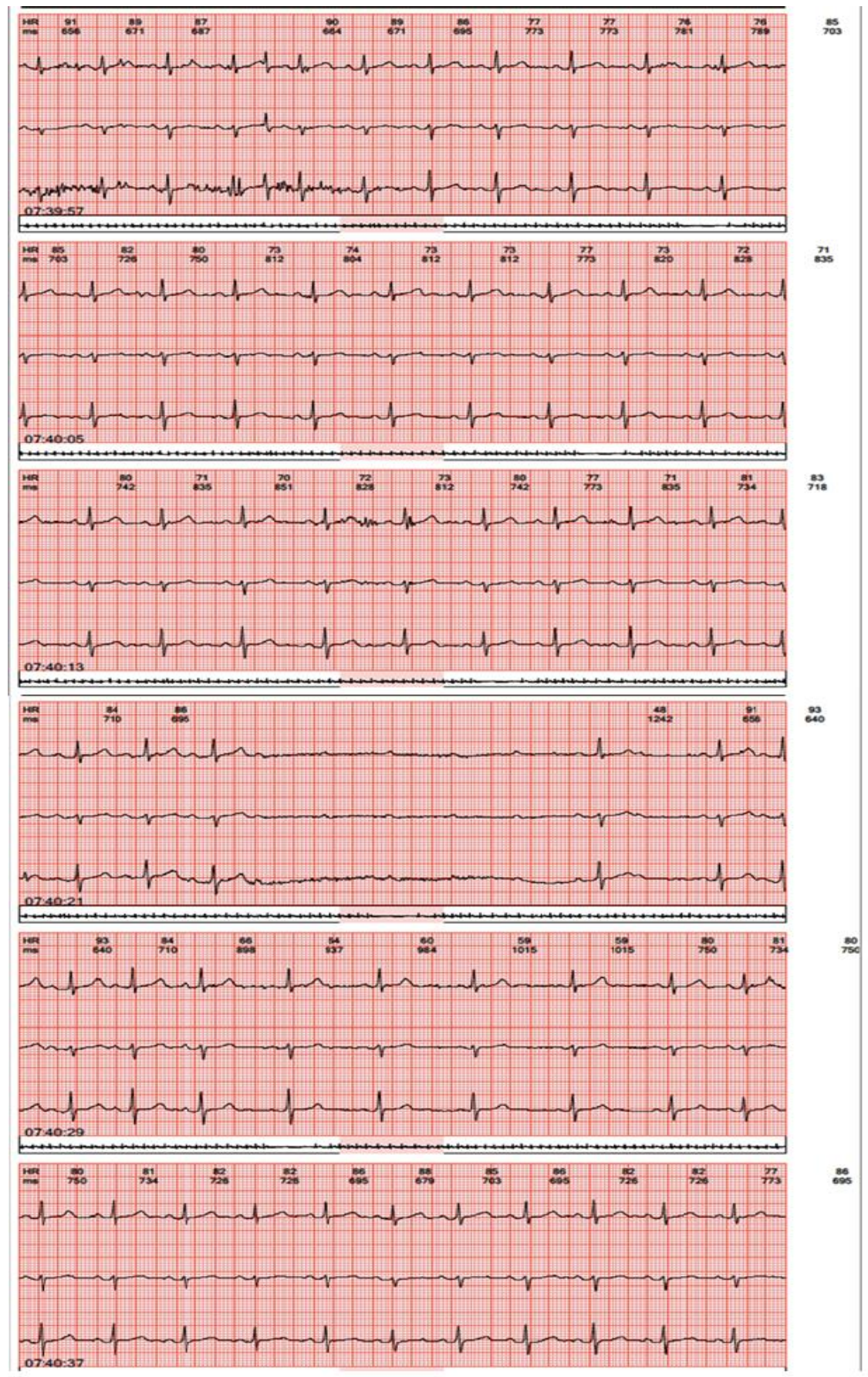

Figura 2. Holter de 24 horas.

Ritmo sinusal de base con adecuado promedio y variabilidad de la frecuencia cardíaca. Sin signos de disfunción sinusal. Repolarización normal. Se documentaron varios episodios de bloqueo aurículoventricular completo paroxístico. A las 7:40 horas se reportó episodio sincopal asociado con el bloqueo aurículo-ventricular completo de 6,12 segundos de duración, el cual recupera en bloqueo aurículo-ventricular 2:1 y finalmente en conducción AV normal. 
No se documentaron alteraciones hidroelectrolíticas o metabólicas, así como tampoco cardiopatía estructural en el ecocardiograma transtorácico. Se realizó Tilt test, el cual fue negativo para síncope neuralmente mediado. Por los hallazgos se consideró candidata a implante de marcapasos bicameral, con una excelente respuesta y sin evidencia posterior de recurrencia del síncope (figura 3 ).

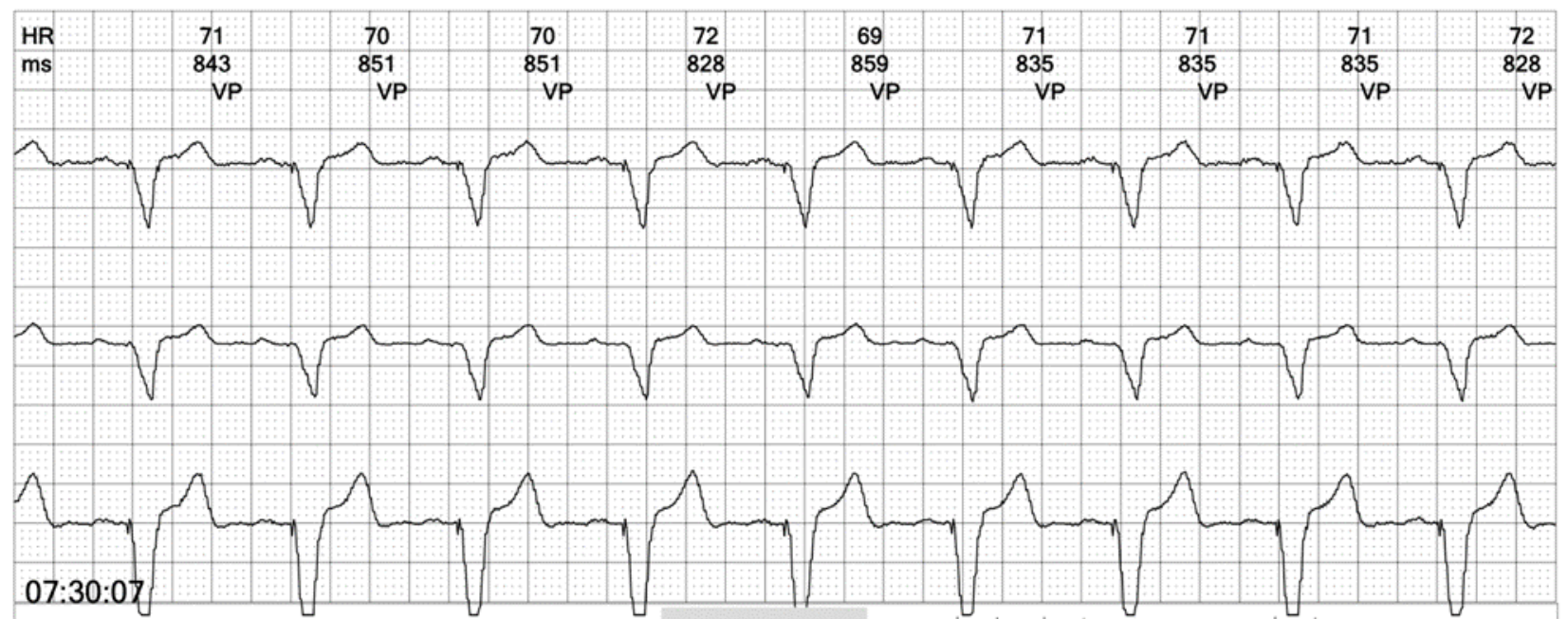

Figura 3. Holter 24 horas.

Control post-implante de marcapaso bicameral programado en modo DDD, en donde se documenta adecuada detección atrial y ventricular y adecuada captura ventricular.

\section{Discusión}

El síncope es una causa frecuente de consulta médica. Se presentan de forma especial en el contexto de bloqueos de la conducción aurículo-ventricular, cuando ocurren de forma súbita y a menudo en pacientes sin cardiopatía isquémica o estructural de base. El síncope es el síntoma principal de los bloqueos aurículo-ventricular hasta en el $40 \%$ de los $\operatorname{casos}^{(11)}$.

El bloqueo aurículo-ventricular completo paroxístico idiopático se caracteriza por episodios sincopales de larga data, ausencia de trastornos en el electrocardiograma o en el estudio electrofisiológico, ausencia de progresión a bloqueo $\mathrm{AV}$ permanente y eficacia con la terapia de estimulación cardíaca. Estos pacientes tienen bajos niveles de adenosina plasmática basal y mayor sensibilidad a la adenosina/ATP exógena ${ }^{(2,12)}$. La fisiopatología ha sido explicada por la presencia de bloqueo en fase 4 del sistema de conducción infraHisiano ${ }^{(11)}$. Es importante anotar 
que esta enfermedad es frecuentemente diagnosticada de forma errónea como un trastorno convulsivo o epilepsia y los pacientes llegan a ser tratados con medicamentos antiepilépticos (ver diagnósticos diferenciales mas importantes en la figura 1$)^{(13)}$.

Brignole et al. $^{(9)}$ realizan un seguimiento a largo plazo de una cohorte de 18 pacientes (nueve hombres y edad promedio de $55 \pm 19$ años) con diagnóstico de síncope secundario a bloqueo aurículo-ventricular completo paroxístico y en quienes no se conocía la etiología que explicara su cuadro. Presentaban además electrocardiograma dentro de límites normales y ausencia de enfermedad cardíaca estructural. Se les realizó monitoreo Holter 24 horas y en todos ellos se documentó bloqueo aurículo-ventricular completo paroxístico durante la presencia de múltiples pausas consecutivas; el bloqueo aurículo-ventricular ocurrió sin prolongación previa del ciclo P-P ni del intervalo PR. Después de que se les implantó el marcapasos cardíaco permanente, ninguno de ellos presentó recurrencias sincopales demostrando, como en el presente caso, la eficacia de la estimulación eléctrica cardíaca.

El bloqueo aurículo-ventricular completo paroxístico fue descrito inicialmente por Sachs y Traynor $^{(14)}$ en 1933, y más tarde por Coumel et al. ${ }^{(15)}$ en 1971. En la literatura se encuentran descritos tres tipos de bloqueo aurículo-ventricular completo paroxístico: Intrínseco: asociado con enfermedad propia del sistema de conducción cardíaca. Los pacientes debutan con crisis de Stokes-Adams y se desencadena frecuentemente por una extrasístole. El electrocardiograma de base suele ser anormal.

Extrínseco vagal: llamado también síncope reflejo; se produce por un influjo parasimpático importante, asociado con pródromos, su electrocardiograma de base suele ser normal, pero presenta un patrón característico, evidenciado con monitoreo electrocardiográfico continuo, cuando se realizan maniobras vagales, incluyendo un intervalo P-P que se prolonga progresivamente con cada nueva longitud de ciclo y una prolongación progresiva del intervalo $\mathrm{PR}$, seguida de un bloqueo aurículo-ventricular completo.

Extrínseco idiopático: asociado con bajos niveles de adenosina plasmática. No presenta pródromos clínicos ni tiene asociado una alteración del ritmo evidente ${ }^{(16)}$.

Es importante determinar el tipo de bloqueo aurículo-ventricular completo paroxístico, principalmente por su tratamiento con dispositivos de estimulación cardíaca, dado que, tanto el bloqueo aurículo-ventricular tipo intrínseco y extrínseco idiopático, tienen buena respuesta al estímulo con marcapasos, mientras en que en el bloqueo aurículo-ventricular extrínseco 
Mayo - agosto de 2021

vagal, la respuesta puede ser variable ${ }^{(1)}$. En el presente caso, al tratarse de una paciente con historia de múltiples episodios sincopales, sin pródromos y con un PR que no varía en el electrocardiograma, se consideró que la causa más probable del síncope fuera un bloqueo aurículo-ventricular completo paroxístico extrínseco idiopático. La excelente respuesta y evolución clínica durante los tres años posteriores al implante del marcapasos bicameral confirmaron la sospecha diagnóstica.

\section{Conclusión}

Existen tres diferentes tipos de bloqueo aurículo-ventricular completo paroxístico. Es importante reconocer el tipo de bloqueo mediante el monitoreo electrocardiográfico y las manifestaciones clínicas, ya que esto define el tratamiento a largo plazo con dispositivo implantable de estimulación eléctrica cardíaca.

\section{Fuente de financiación}

Ninguna.

\section{Conflicto de Interés}

Se declara que no existe ningún conflicto de interés en el presente artículo.

\section{Ética de la publicación}

Se trata de un reporte de caso, que no incluye experimentación con animales ni ensayos clínicos.

\section{Bibliografía}

1. Shen Win-Kuang, Sheldon Robert S., Benditt David G., Cohen Mitchell I., Forman Daniel E., Goldberger Zachary D. et al. 2017 ACC/AHA/HRS Guideline for the evaluation and management of patients with syncope: a report of the American College of Cardiology/American Heart Association Task Force on Clinical Practice Guidelines and the Heart Rhythm Society. Circulation. 2017;136 (5):e60-122.

2. Brignole M, Moya A, De Lange FJ, Deharo JC, Elliott PM, Fanciulli A, et al. 2018 ESC Guidelines for the diagnosis and management of syncope. European Heart Journal 2018;39, 1883-1948. 
3. Blanc J-J. Syncope: Definition, Epidemiology, And Classification. Cardiol Clin. 2015;33 (3):341-5.

4. Runser LA, Gauer RL, Houser A. Syncope: evaluation and differential diagnosis. Am Fam Physician. 2017;95 (5):303-12.

5. Martow E, Sandhu R. When is syncope arrhythmic? Med Clin North Am. 2019103 (5):793-807.

6. Thiruganasambandamoorthy V, Hess EP, Turko E, Tran M-L, Wells GA, Stiell IG. Defining abnormal electrocardiography in adult emergency department syncope patients: the Ottawa Electrocardiographic Criteria. Can J Emerg Med. 2012;14 (4):252-62.

7. Lee S, Wellens HJJ, Josephson ME. Paroxysmal atrioventricular block. Heart Rhythm. 2009;6 (8):1229-34.

8. Alboni P, Holz A, Brignole M. Vagally mediated atrioventricular block: pathophysiology and diagnosis. Heart Br Card Soc. 2013;99 (13):904-8.

9. Brignole M, Deharo J-C, De Roy L, Menozzi C, Blommaert D, Dabiri L, et al. Syncope due to idiopathic paroxysmal atrioventricular block: long-term follow-up of a distinct form of atrioventricular block. J Am Coll Cardiol. 2011;58 (2):167-73.

10. Bansal R, Mahajan A, Rathi C, Mehta A, Lokhandwala Y. What is the mechanism of paroxysmal atrioventricular block in a patient with recurrent syncope? J Arrhythmia. 2019;35 (6):870-2.

11. Menon SMD, Ribas CS, Meneclier CAR, Morillo CA. Intermittent atrioventricular block: What is the mechanism? Heart Rhythm. 2012;9 (1):154-5.

12. Brignole M, Gaggioli G, Menozzi C, Gianfranchi L, Bartoletti A, Bottoni N, et al. Adenosineinduced atrioventricular block in patients with unexplained syncope: the diagnostic value of ATP testing. Circulation. 1997;96 (11):3921-7.

13. Cai SY, Ye SF, Wu X, Xiang MX, Wang JA. Torsade de pointes in a patient with complete atrioventricular block and pacemaker failure, misdiagnosed with epilepsy. J Electrocardiol. 2015; 48(3):450-454. 
14. Sachs A, Traynor RL. Paroxysmal complete auriculo-ventricular heart-block: A case report. Am Heart J. 1933 1;9 (2):267-71.

15. Coumel P, Fabiato A, Waynberger M, Motte G, Slama R, Bouvrain Y. Bradycardiadependent atrio-ventricular block: Report of two cases of A-V block elicited by premature beats. J Electrocardiol. 1971;4 (2):168-77.

16. Brignole M, Deharo J-C, Guieu R. Syncope and Idiopathic (Paroxysmal) AV Block. Cardiol Clin. 2015;33 (3):441-7. 